\title{
Screening key genes related to ovarian cancer and exploring their possible molecular mechanisms
}

\author{
Yuehua Zhang \\ Xiamen University \\ Zhixiong Huang \\ Xiamen University \\ Bin Zhao \\ Xiamen University \\ Ke Huang \\ Guangxi Medical University \\ Xingyan Li \\ Guangxi Medical University \\ Qionghua Chen \\ Xiamen University
}

Junzui Li ( $\nabla$ linchuangkeyan@yeah.net )

Xiamen University https://orcid.org/0000-0002-6640-8215

\section{Research article}

Keywords: Ovarian cancer, GEO data, Differentially Expressed Genes, Integrated bioinformatics, GO analysis

Posted Date: October 23rd, 2019

DOI: https://doi.org/10.21203/rs.2.16321/v1

License: (c) (i) This work is licensed under a Creative Commons Attribution 4.0 International License.

Read Full License 


\section{Abstract}

Background As one of the common malignant tumors in women, ovarian cancer (OC) often exerts the atypically early clinical symptoms. Therefore, it is particularly important for seeking more effectively early diagnosis of OC (biomarkers). Besides, although a lot of sequencing and chip research have been done on the pathogenesis of $\mathrm{OC}$, the pathogenesis, clinical and genetic features of $\mathrm{OC}$ is still not very clear.

Methods In this study, 4 GEO data (GSE66957, GSE119054, GSE14407 and GSE54388) were selected for differential expression gene analysis (DEGs), and the important template of the 4 DEGS overlapping genes was taken as Hub genes. Then, the GO and pathway enrichment analysis were conducted to confirm the enrichment of these Hub genes, and these Hub genes were identified as key genes. In addition, the transcriptional levels of these Hub genes in $\mathrm{OC}$ and their impacts on the overall survival rate of OC were validated via the UCSC and TCGA datasets.. Besides, cBioPortal, TargetScan, UCSC, DiseaseMeth and TIMER software were performed to explore the potential biological functions of these key genes in $\mathrm{OC}$.

Results We screened out 10 Hub genes related to OC including VEGFA, ZWINT, CDKN2A, SLC2A1, TOP2A, MKI67, CCND1, KPNA2, FGF2 and SMC4, and further demonstrated that they were most significantly enriched in protein binding, cytoplasm, nucleus, extracellular exosome, membrane, cell division, cell adhesion and pathways in cancer. Meanwhile, CCND1, TOP2A, SMC4 and FGF2 were screened out as key candidate genes associated with $\mathrm{OC}$. Further analysis proved these key candidate genes may regulate the occurrence and development of $\mathrm{OC}$ through mediating the gene mutation, miRNAs and genetic epigenetics such as methylation and acetylation.

Conclusion These data would improve our understanding of the causes and underlying molecular events of $\mathrm{OC}$, be of clinical significance for the early diagnosis and prevention of $\mathrm{OC}$, and may provide the promising therapeutic targets in OC.

\section{Background}

$\mathrm{OC}$ is one of the common malignant tumors in gynecological diseases, accounting for $5 \%$ of female malignant tumors, and its incidence ranks third only after cervical cancer and uterine body cancer. There are still more than 14,000 women suffering from OC every year [. Given its deep location (deep pelvic cavity) and atypical symptoms, it is difficult to detect OC in time []. Recently, although researchers have made extensive progress in the treatment of $\mathrm{OC}[$,the prevention and prognosis of $\mathrm{OC}$ are still frustrating 7. According to the progress of oncology medical treatment in 2017, less than half of the newly diagnosed $O C$ patients survived for more than 5 years $\rrbracket$, which is mainly due to the patient's visiting time, for most patients have been in advanced cancer at the time of visiting. Therefore, the early diagnosis of $\mathrm{OC}$ is still an important and urgent problem for women's health.

Nowadays, scientists have uploaded all gene data related to OC to GEO and TCGA databases with the rapid development of gene chip, transcript sequencing and large data analysis [. In addition, increasing 
evidences show that the mutations and abnormal expressions of genes including SIRT1, HBA2, BRCA, ZWINT, SPP1 and HBG2 are related to the occurrence and development of OC[3,,]. However, these biomarker genes still do not have a good early diagnostic effect in clinical practice, and the incidence and mortality of $\mathrm{OC}$ are still high [. Therefore, it is essential to understand the precise molecular biological mechanisms involved in the occurrence, proliferation and recurrence of $\mathrm{OC}$ and formulate the effective diagnosis and treatment strategies, thereby preventing the $\mathrm{OC}$ occurrence and reducing the $\mathrm{OC}$ mortality. In this study, we screened out Hub genes that might be involved in the development of OC via using a series of bioinformatics analysis and explored the potential biological process of these key candidate genes in OC.

The workflow of this study is divided into seven main steps: select 4 raw microarray datasets (GSE66957, GSE119054, GSE14407 and GSE54388) related to OC from the GEO datasets (https://www.ncbi.nlm.nih.gov/geo/) for differential expression gene analysis (DEGs) , analyze the Gene Ontology (GO) and Kyoto Encyclopedia of Genes and Genomes (KEGG) pathway enrichment of overlapping genes of the 4 DEGS via DAVID (https://david.ncifcrf.gov/), construct the Protein-Protein Interaction (PPI) network using STRING online software (https://string-db.org/) and Cytoscape to analyze the correlation among these common DEGs, screen out the 10 Hub genes related to $O C$, validate the survival of $\mathrm{OC}$ and the transcriptional levels of these 10 hub genes in $\mathrm{OC}$ by cBioPortal(http://www.cbioportal.org/) and GEPIA (http://gepia.cancer-pku.cn/), respectively, seek for the potential key candidate genes, which are related to the occurrence and development of OC. use the TargetScan (http://www.targetscan.org/vert_72/), TIMER (https://cistrome.shinyapps.io/timer/), UCSC (http://genome.ucsc.edu/index.html) and DiseaseMeth (http://biobigdata.hrbmu.edu.cn/diseasemeth/index.html) to explore the potential function of targeting miRNAs, gene mutation, acetylation and methylation of these key genes and their relationship with tumor immune infiltration.

In conclusion, we screened out the common DEGs, the Hub genes and even the key candidate genes through a series of bioinformatics methods. Meanwhile, the potential molecular and molecular mechanisms involved in the regulation of OC development are further explored on the basis of TCGA and UCSC databases, which would improve our understanding of its causes and the underlying molecular events. These candidate genes may provide a promising future for the research of therapeutic targets in OC.

\section{Methods And Results}

\section{GEO data collection related to OC}

We explored the microarray datasets differentially expressed between human OC tissues and ovarian normal tissues by searching the keywords including ovarian cancer, ovarian tumor and ovarian carcinoma from the GEO database which is one of the largest and most comprehensive public gene expression databases. Raw microarray datasets, GSE66957(Last update date, May 07, 2018)【, 
GSE119054(Last update date, Mar 20, 2019)[], GSE14407(Last update date, Mar 25, 2019)], and GSE54388 (Last update date, Mar 25, 2019)] which contained a total of 134 samples with 91 cases of OC samples and 43 cases of normal ovarian samples $\bigotimes$ Table $1 \rrbracket$ were selected and the gene expression of each microarray is shown in Fig. 1B.

\section{Screening of common DEGs}

We divided each of microarray datasets into malignant tumor group and normal group, and screened out DEGs between 2 groups of microarray datasets by GEO2R(https://www.ncbi.nlm.nih.gov/geo/geo2r/) which is an online data analysis software that automatically screens for DEGs between two sets of chip data on the GEO database. The DEGs between the 2 groups were considered statistically significant with adjusted $P$ values $<0.05$ and $\mid \log 2$ fold change (FC) $\mid \geq 1$ (Table 2). Cluster analysis of gene expression of the first 100 differentially expressed genes (50 up and 50 down) between the 2 groups is shown in Fig. 2 (plotted by R). Subsequently, we screened out 103 common DEGs (73 up and 30 down) by Veen Diagrams online software (http://bioinformatics.psb.ugent.be/webtools/Venn/) (Table 3 and Fig. 3).

Go and KGG pathways enrichment analysis of common DEGs

103 common DEGs were poured into the DAVID, a classic gene enrichment analysis site for the analysis of DEGs functions and pathway enrichment, for GO and KEGG pathway analysis, and the results with $P<0.05$ were selected. We divided the output of the results into 4 functional categories: biological process (BP), cellular component (CC), molecular function and KEGG pathway (Table4 and Fig. 4). GO enrichment analysis showed that the common DGEs were mostly enriched in protein binding, cytoplasm, nucleus, extracellular exosome, membrane, cell division and cell adhesion. Moreover, the first 10 biological processes of obvious enrichment are all associated with the up-regulated genes, but not the downregulated genes which were only enriched in nervous system development, cell differentiation and somatic stem cell population maintenance. In addition, KEGG pathway enrichment analysis showed that the common DEGs were largely existed in p53 signaling pathway $s$ in bladder cancer and pancreatic cancer. Combined with the above results, we found that up-regulated common DEGs were enriched in these pathways, while the down-regulated differential genes do not have significant signaling pathway enrichment.

\section{Screening of Hub genes}

We imported these 103 common DEGs into STRING and Cytoscapte to construct the Protein-Protein Interaction (PPI) network (Fig. 5A). We calculated the top 15 genes using the 12 algorithms (Betweenness, Closeness, Clustring, Degree, DMNC, EcCentricity, EPC, MCC, MNC, Radiality, Stres, and Radiality) that come with the CytoHubba plug-in. In addition, we took the overlapping genes as Hub genes, including VEGFA, ZWINT, CDKN2A, SLC2A1, TOP2A, MKI67, CCND1, KPNA2, FGF2 and SMC4. These 10 Hub genes exhibited the high expression except for MKI67 and FGF2 in OC (Fig. 5B). 
The $10 \mathrm{Hub}$ genes were introduced into cBioPortal, a TCGA database online analysis software, to explore their co-expression and network in OC (Fig. 5C). Transcriptional levels of Hub genes and the related different tumor grades based on the TCGA database were validated by GEPIA (Fig. 6). The results showed that only FGF2 was lowly expressed and the other 9 Hub genes were highly expressed in OC, which was consistent with the results obtained from the GEO database. The transcriptional levels of SLC2A1, VEGFA and ZWINT in different grades of $\mathrm{OC}$ is different, while the expression of the other genes showed no significant difference in different levels of OC. Moreover, we studied the effect of these Hub genes on the overall survival rate of OC. There are 4 key candidate genes including CCND1 (up), TOP2A (up), SMC4 (up) and FGF2 (down) related to survival and prognosis of OC were screened out (Fig. 7).

\section{Function of key candidate genes and its regulation mechanism of OC}

At this point, our study has identified 4 key candidate genes for OC. However, why do these key candidate genes change significantly? or through which molecular biological mechanisms do they regulate the occurrence of OC? None of these mechanisms is clear. More in-depth study is carried out to explore the potential molecular and biological mechanisms of key candidate genes in the development of OC. The gene expression levels of multiple tumors obtained from GEPIA indicated that CCND1 was highly expressed in BRCA (Breast Cancer), KIRC (Kidney Renal Clear Cell Carcinoma) and OV, while TOP2A was highly expressed in BRCA, LUSC (Lung Squamous Cell Carcinoma) and OV. Meanwhile, SMC4 is highly expressed in BRCA, CESC (Cervical Squamous Cell Carcinoma and Endocervical Adenocarcinoma) and OV. However, FGF2 is down-regulated in LUAD and OV (Fig. 8A). Interestingly, a one-to-one correlation study between FGF2 and CCND1, TOP2A and SMC4 showed that they were not related to each other (Fig. 8B).

To further explore through which epigenetics way these key candidate genes were differentially expressed in $\mathrm{OC}$, we performed $\mathrm{CBioPortal}$ to understand the proportion of genes mutation types and clustering expression of the 4 key candidate genes in OC. These key candidate genes exhibited different gene mutation types and degrees in sequence. The results showed that there was no mutation in CCND1, but the other three key genes had mutations, in which the most common mutation was missense mutation (Fig. 8C). Moreover, we used TIMER, UCSC and DiseaseMeth software to analyze the mechanisms of tumor immune infiltration, acetylation and methylation of these genes, respectively. Results demonstrated that there was no significant correlation between these key gene expressions and tumor immune infiltration (Fig. 9A, ploted by TIMER). The epigenetic regulation (acetylation, methylation and IncRNA) of these genes were subsequently detected through the UCSC database. The results indicated that all these 4 key genes had the methylation and acetylation sites (Fig. 9B and Fig. 10A). However, there was no enrichment of IncRNA around the sequences of these 4 key candidate genes. Surprisingly, the methylation levels of CCND1, TOP2A and SMC4 in OC were higher than that in normal, while the methylation levels of FGF2 showed the opposite result (Fig. 10B, ploted by DiseaseMeth). What's more, CCND1, TOP2A, SMC4 and FGF2 combined with miR-142-5p, miR-144-3p, miR-219-5p and miR23-3p, respectively (Fig. 11, ploted by TargetScan). 


\section{Discussion}

$\mathrm{OC}$ is one of the most common malignant tumors in female reproductive organs, and its incidence ranks third only after cervical cancer and uterine body cancer. Currently, OC is divided into four grades according to FIGO (International Federation of Obstetrics and Gynecology), and each grade is classified according to the location and status of the tumors []. Although this classification can guide the treatment and prognosis of OC],the etiology and pathogenesis of OC are still unclear [. Meanwhile, given that its clinical symptoms are atypical, it is difficult to get early diagnosis and treatment, and it seriously affects the quality of life of female patients. Some studies have pointed out the overall treatment of $\mathrm{OC}$ is unsatisfactory, such as $80 \%$ of patients with OC are advanced patients, $70 \%$ of patients will relapse [. It's remarkable that one third of patients with advanced OC cannot live for 5 years []. Therefore, the early diagnosis of $\mathrm{OC}$ is still a difficult problem. Screening key genes related to OC by integrated bioinformatics and exploring their possible biological processes will promote the development of early diagnosis of OC, and may improve the prognosis of $\mathrm{OC}$ and the quality of patients' life.

Previous studies have shown that multiple signaling pathways and tumor immune infiltration regulate the progression of OC []. For example, Huang et al. have pointed out high CD8+lymphocytes density in tumor tissues is significantly associated with the improved progression free survival in high-grade serous OC []. Besides, many gene mutations, epigenetic regulation, transcriptional regulation and translation modification have also been reported to be associated with the progression of OC [. However, most cases of $\mathrm{OC}$ without an early finding are not candidates for curative therapies, which may be one of the reasons for poor patient prognosis. Thus, it is urgent for seeking the potential markers for diagnosis and treatment with high efficiency. Microarray technology enables us to explore the genetic alterations in $\mathrm{OC}$, and has been proved to be a useful approach to identify new biomarkers in other diseases.

In this study, we combined with GEO database, TCGA data and a variety of comprehensive bioinformatics methods to screen out the key genes related to $O C$ and further explore the possibly related molecular biological mechanisms. Through analyzing GEO2R, GO and KEGG pathways, we found that VEGFA, ZWINT, CDKN2A, SLC2A1, TOP2A, CCND1, KPNA2, and SMC4 were significantly up-regulated in OC, while MKI67 and FGF2 were significantly down-regulated. Meanwhile, these genes mainly functioned in protein binding, cytoplasm, nucleus, extracellular exosome, membrane, cell division and cell adhesion. Moreover, we further proved that CCND1, TOP2A, SMC4 and FGF2 might regulate the occurrence of OC through miRNAs, gene mutations, acetylation (H3K4Me and H3K27AC), methylation through searching for TCGA, UCSC, TIMER and TargetScan software. In our research, although these key genes have nothing to do with IncRNA, IncRNA may be involved in ovarian cancer through other genes[]. As we known, CCND1, a proto-oncogene associated with cell division cycle, has been reported to be related with breast cancer, bladder cancer, parathyroid cancer, lymphoma, and lung cancer[, and it also promotes the cell proliferation via mediating the transition from $\mathrm{G} 1$ phase to $\mathrm{S}$ phase of cell cycle. At present, some studies have also shown that CCND1 participates in regulation of OC []. CCND1 silencing could break DNA double strands [, thus inhibiting the growth of OC. However, the mechanism is still unknown. Therefore, the 
mutation, methylation and acetylation of genes that we have analyzed through bioinformatics would provide a certain degree of reference value for further in-depth study.

Topoisomerase (DNA) II alpha (TOP2A) could convert DNA superhelix into a relaxed state. Previous studies have confirmed that TOP2A is generally overexpressed in OC tissues, and they speculate that TOP2A may be functioned as a potential biomarker []. A new study also shows that TOP2A can be used as a marker of PEG-Lyposomal doxorubicin (PLD) response in epithelial OC [], and can also participate in the p53 signaling pathway to coordinate the occurrence of various malignant tumors [. However, the mechanism of TOP2A regulating $\mathrm{OC}$ is still unknown, and phenotypic studies are still in progress. Here, we proved that it would be useful for researchers studying TOP2 through the methylation and acetylation.. It is well known that SMC4, a structural maintenance of chromosomes, is an important component of the core catalytic group of condensin. The main role of condensin is to regulate the dynamic changes of chromosomes during cell cycle. However, the in vivo conformation and molecular mechanism of the heterodimers formed by SMC4 in condensers are still unclear. Recent studies have demonstrated that mutation of SMC4 gene further affects chromosomal stability after the change of condensin, further leading to the occurrence of various cancers, such as breast cancer and colorectal cancer. Meanwhile, SMC4 can bind with SMC2 to form SMC2/SMC4 heterodimer structure (the core catalytic group of condensers). However, there is no similar research report involved in oophoroma. FGF2 has been reported to have an effective angiogenesis effect in vivo and in vitro $\Delta$ through stimulating the growth of smooth muscle cells and promoting wound healing and tissue regeneration. Besides, it may also play an important role in the differentiation and function of the nervous system and in the regeneration of eyes and bones []. Recent studies have revealed that miRNA-936 targets FGF2 to suppress the epithelial OC aggressiveness by deactivating the PI3K/Akt pathway. Consistent with this report, we also found that FGF2 could target miRNAs to regulate the occurrence of $\mathrm{OC}$, but the reported miRNA-936 and PI3K/Akt pathway are not the key factors for the role of FGF2.

On the other hand, GO and KEGG pathway enrichment analysis results showed that the common differential genes were mostly significant enriched in protein binding, cytoplasm, nucleus, extracellular exosome, membrane, cell division, cell adhesion, pathways in cancer, bladder cancer, pancreatic cancer, and p53 signaling pathway. Combining with the recent theories that $\mathrm{OC}$ exosomes can act as coordinators of pre-metastatic niche formation, biomarkers suitable for liquid biopsy and targets for chemotherapy [,], it is very important to identify whether these key genes exist in the OC exosomes. As we know, P53 mutations are associated with poor prognosis, because that P53 can suppress cancer progression by inducing cell cycle arrest or apoptotic process, and can respond to a variety of cell stress signals. Based on these data, we propose that CCND1, TOP2A, SMC4 and FGF2 may regulate the occurrence of $O C$ through miRNA, gene mutation and epigenetic (methylation and acetylation). Unfortunately, there's still no research carried out to explore the regulatory role of these key candidate genes in OC.

It is particularly noteworthy the 4 key candidate genes acetylation. UCSC results showed that CCND1, TOP2A, SMC4 and FGF2 had H3K4Me and H3K27AC acetylation sites in our study. Previous studies have 
shown that H3K4Me acetylation of these genes can up-regulate the expression of BIRC3, thereby further inhibiting the growth of $\mathrm{OC}$ by promoting cell apoptosis and inhibiting proliferation [. Based on these data, CCND1/TOP2A/SMC4/FGF2-H3K4Me-BIRC3 pathway may be involved in OC.

\section{Conclusions}

In this study, 103 common DEGs related to OC were screened out in GEO database via using R software and bioinformatics analysis. The enrichment analysis of GO and KEGG pathway showed that these DEGs mostly significant enriched in protein binding, cytoplasm, nucleus, extracellular exosome, membrane, cell division, cell adhesion, pathways in cancer, bladder cancer, pancreatic cancer and p53 signaling pathway, which would provide a theoretical basis for studying the biological processes of OC. Then, we screened out 10 Hub genes by comprehensive bioinformatics analysis and successfully selected 4 key candidate genes related to $\mathrm{OC}$ via the survival analysis and the transcription level analysis. Finally, we explored the mechanisms of these 4 key candidate genes through various online software. Further exploration would be beneficial for understanding the interaction among these 4 key candidate genes, which might regulate the development of OC through various molecular biological mechanisms such as gene mutation, regulation of targeted miRNAs, acetylation and methylation of these key candidate genes.

The results of this study would improve our understanding of the pathogenesis of $\mathrm{OC}$ and the occurrence and development of the underlying molecular mechanisms. These findings might also have important clinical significance for the early diagnosis $₫$ treatment and the prevention of OC\as well as be helpful for subsequent experimental studies to find potential key molecule targets of OC. However, these results are derived from large data analysis, which need to be further confirmed by more direct experimental studies.

\section{Declarations}

\section{Acknowledgements}

Not applicable

\section{Funding}

This study was supported by the National Science Foundation of China (No. 8187060862). The funder had no role in study design, data collection and analysis, except for bioinformatics training, writing the manuscript, and decision to publish.

\section{Availability of data and materials}

The expression data associated with this article is available on GEO databases(https://www.ncbi.nlm.nih.gov/geo/). 


\section{Authors' contributions}

JZLperformed comparative analysis using bioinformatics tools. $\mathrm{ZXH}, \mathrm{KH}, \mathrm{XYL}$ and $\mathrm{BZ}$ participated in data analysis and discussion. JZL and YHZ interpreted data and wrote the manuscript. QHC organized and supervised the project. All authors read and approved the final manuscript.

Ethics approval and consent to participate

Not applicable

Consent for publication

Not applicable

\section{Competing interests}

The authors declare that they have no competing interests.

\section{References}

1. Matsuo K, Cripe JC, Kurnit KC, et al. Recurrence, death, and secondary malignancy after ovarian conservation for young women with early-stage low-grade endometrial cancer. Gynecol Oncol. 2019.

2. Zhong PP, Zhu L, Zhang LH, et al. [Clinicopathological features of ovarian Brenner tumors]. Zhonghua Bing Li Xue Za Zhi. 2019;48(8):615-619.

3. Salama M, Anazodo A, Woodruff TK. Preserving fertility in female patients with hematological malignancies: A multidisciplinary oncofertility approach. Ann Oncol. 2019

4. Phillips $A$, Kehoe $S$, Singh $K$, et al. Socioeconomic differences impact overall survival in advanced ovarian cancer (AOC) prior to achievement of standard therapy. Arch Gynecol Obstet. 2019.

5. Schwartzberg L, Kim ES, Liu D, et al. Precision Oncology: Who, How, What, When, and When Not?. Am Soc Clin Oncol Educ Book. 2017.

6. Zhao B, You Y, Wan Z, et al. Weighted correlation network and differential expression analyses identify candidate genes associated with BRAF gene in melanoma[J]. BMC Medical Genetics, 2019, 20(1).

7. Bu H, Chen J, Li Q, et al. BRCA mutation frequency and clinical features of ovarian cancer patients: A report from a Chinese study group. J Obstet Gynaecol Res. 2019.

8. Nakamura K, Sawada K, Kobayashi M, et al. Role of the Exosome in Ovarian Cancer Progression and Its Potential as a Therapeutic Target. Cancers (Basel). 2019;11(8).

9. Berzina D , Nakazawa-Miklasevica M , Zestkova J , et al. BRCA1/2 mutation screening in high-risk breast/ovarian cancer families and sporadic cancer patient surveilling for hidden high-risk families[J]. BMC Medical Genetics, 2013, 14(1):61. 
10. Gourley C, Bookman MA. Evolving Concepts in the Management of Newly Diagnosed Epithelial Ovarian Cancer. J Clin Oncol. 2019.

11. Xiao Y , Shaoming Z , Li L , et al. Identification of differentially expressed genes and signaling pathways in ovarian cancer by integrated bioinformatics analysis[J]. OncoTargets and Therapy, 2018, Volume 11:1457-1474.

12. Dong $\mathrm{S}$, Wang $\mathrm{R}$, Wang $\mathrm{H}$, et al. HOXD-AS1 promotes the epithelial to mesenchymal transition of ovarian cancer cells by regulating miR-186-5p and PIK3R3[J]. Journal of Experimental \& Clinical Cancer Research, 2019, 38(1).

13. Bowen $N \mathrm{~J}$, Walker $D E$, Matyunina $L V$, et al. Gene expression profiling supports the hypothesis that human ovarian surface epithelia are multipotent and capable of serving as ovarian cancer initiating cells[J]. BMC Medical Genomics, 2009, 2(1):71.

14. Yeung $T L$, Leung $C S$, Wong $K K$, et al. ELF3 is a negative regulator of epithelial-mesenchymal transition in ovarian cancer cells[J]. Oncotarget, 2017, 8(10):16951-16963.

15. Wang R, Tao X, Wu X, et al. The Number of Removed Pelvic Lymph Nodes as a Prognostic Marker in FIGO Stage IB1 Cervical Cancer with Negative Lymph Nodes. J Minim Invasive Gynecol. 2019.

16. Berzina $D$, Nakazawa-Miklasevica $M$, Zestkova J , et al. BRCA1/2 mutation screening in high-risk breast/ovarian cancer families and sporadic cancer patient surveilling for hidden high-risk families[J]. BMC Medical Genetics, 2013, 14(1):61.

17. Han Y, Kim B, Cho U, et al. Mitochondrial fission causes cisplatin resistance under hypoxic conditions via ROS in ovarian cancer cells. Oncogene. 2019.

18. US Preventive Services Task Force., Owens DK, Davidson KW, et al. Risk Assessment, Genetic Counseling, and Genetic Testing for BRCA-Related Cancer: US Preventive Services Task Force Recommendation Statement. JAMA. 2019;322(7):652-665.

19. Antony F, Deantonio $C$, Cotella $D$, et al. High-throughput assessment of the antibody profile in ovarian cancer ascitic fluids. Oncoimmunology. 2019;8(9):e1614856.

20. Liu YL, Zhou Q, lasonos A, et al. Subsequent therapies and survival after immunotherapy in recurrent ovarian cancer. Gynecol Oncol. 2019.

21. Huang $X M$, Zhang $Y, X u L$, Wang $M$, Wang $W$. [Clinical significance of tumor infiltrating lymphocytes in high-grade serous ovarian carcinoma]. Zhonghua Bing Li Xue Za Zhi. 2019;48(8):610-614.

22. Parmar K, Kochupurakkal BS, Lazaro JB, et al. The CHK1 Inhibitor Prexasertib Exhibits Monotherapy Activity in High-Grade Serous Ovarian Cancer Models and Sensitizes to PARP inhibition. Clin Cancer Res. 2019.

23. Zhao Q , Fan C . A novel risk score system for assessment of ovarian cancer based on co-expression network analysis and expression level of five IncRNAs[J]. BMC Medical Genetics, 2019, 20(1).

24. Yan H, Li H, Silva MA, et al. LncRNA FLVCR1-AS1 mediates miR-513/YAP1 signaling to promote cell progression, migration, invasion and EMT process in ovarian cancer. J Exp Clin Cancer Res. 2019;38(1):356. 
25. Chang W, Wang Y, Li W, et al. MicroRNA-551b-3p inhibits tumour growth of human cholangiocarcinoma by targeting Cyclin D1. J Cell Mol Med. 2019;23(8):4945-4954.

26. Zheng B, Liu F, Zeng L, et al. Overexpression of Pyruvate Kinase Type M2 (PKM2) Promotes Ovarian Cancer Cell Growth and Survival Via Regulation of Cell Cycle Progression Related with Upregulated CCND1 and Downregulated CDKN1A Expression. Med Sci Monit. 2018.

27. Zhong Q, Hu Z, Li Q, et al. Cyclin D1 silencing impairs DNA double strand break repair, sensitizes BRCA1 wildtype ovarian cancer cells to olaparib. Gynecol Oncol. 2019;152(1):157-165.

28. Bai Y, Li LD, Li J, et al. Targeting of topoisomerases for prognosis and drug resistance in ovarian cancer. J Ovarian Res. 2016;9(1):35.

29. Ghisoni E, Maggiorotto F, Borella F, et al. TOP2A as marker of response to pegylated lyposomal doxorubicin (PLD) in epithelial ovarian cancers[J]. Journal of Ovarian Research, 2019, 12(1).

30. Bar JK, Grelewski P, Noga L, et al. The association between the p53/topoisomerase I and p53/ topoisomerase llalpha immunophenotypes and the progression of ovarian carcinomas. Adv Clin Exp Med. 2012;21(1):35-42.

31. Sun $Y$, Cheng $Y$, Zhang $Y$, et al. MicroRNA-889-3p targets FGFR2 to inhibit cervical cancer cell viability and invasion. Exp Ther Med. 2019;18(2):1440-1448.

32. Fenwick A L , Goos J , Rankin J , et al. Apparently synonymous substitutions inFGFR2affect splicing and result in mild Crouzon syndrome[J]. BMC Medical Genetics, 2014, 15(1):95.

33. Ciepliński K, Jóźwik M, Semczuk-Sikora A, et al. Expression of p53 and selected proliferative markers (Ki-67, MCM3, PCNA, and topoisomerase Ila) in borderline ovarian tumors: Correlation with clinicopathological features. Histol Histopathol. 2018;33(2):171-179.

34. Feng W, Dean DC, Hornicek FJ, et al. Exosomes promote pre-metastatic niche formation in ovarian cancer. Mol Cancer. 2019;18(1):124.

35. Nakamura K, Sawada K, Kobayashi M, et al. Role of the Exosome in Ovarian Cancer Progression and Its Potential as a Therapeutic Target. Cancers (Basel). 2019;11(8)

36. Jiang Y, Lyu T, Che X, et al. Overexpression of SMYD3 in Ovarian Cancer is Associated with Ovarian Cancer Proliferation and Apoptosis via Methylating H3K4 and H4K20. J Cancer. 2019;10(17):40724084.

\section{Tables}

Table 1 Details for GEO ovarian cancer data

\begin{tabular}{ccc}
\hline GEO & Normal ovarian tissue & Ovarian cancer tissue \\
\hline GSE66957 & 12 & 57 \\
GSE119054 & 3 & 6 \\
GSE14407 & 12 & 12 \\
GSE54388 & 6 & 16 \\
Total & 43 & 91 \\
\hline
\end{tabular}


Table 2 Number of DEGs

\begin{tabular}{cccc}
\hline GEO & Upregulated & Downregulated & All \\
\hline GSE66957 & 2799 & 1516 & 4315 \\
GSE119054 & 1654 & 1003 & 2657 \\
GSE14407 & 3498 & 2159 & 5657 \\
GSE54388 & 1944 & 1244 & 3188 \\
\hline
\end{tabular}

Table 3 Screening common DEGs in OC by integrated microarray

\begin{tabular}{cl}
\hline \multicolumn{1}{c}{ Degs } & \multicolumn{1}{c}{ Gene names } \\
\hline Upregulated(73) & CDH6 FLAD1 STC2 SLC44A2 F11R CASZ1 CXXC5 SLC2A1 \\
& CDKN2A SORT1 CCDC88C NUAK2 EFHC1 BACE2 CCDC6 AIF1L \\
& EMB LPAR5 MYO6 GALNT6 PNISR ARL4C TPX2 SHMT2 TTC39A \\
& VEGFA DZIP3 CCND1 COL4A1 CDON RHOF VANGL1 KPNA2 \\
& MAGI1 GCNT1 N4BP2L1 ZNF48 STRBP CENPO ZWINT BID \\
& ETNK1 SLC25A27 NDE1 TOP2A SMC4 TMEM8B CFL1 PSD4 HN1 \\
& DNAJC5 ETV6 SUSD2 PFKP LAMA2 HPSE N4BP2L2 ARPC5L \\
& L3MBTL3 NAA38 COMMD5 PSMD2 CMTM4 JMJD1C CTTN DST \\
& CHD7 RTN1 ACER3 CABLES1 CIRBP TOMM5 PDIA4 \\
\hline Downregulated(30) & MKI67 ARX ATP11A PCDH7 NEFH CXADR FOXN3 NPHP3 PRTG \\
& SPC24 SIGLEC11 CMTM7 RBMS3 CCDC68 TBC1D4 NEGR1 DLG2 \\
& NR3C2 FGF2 CRLS1 CLDN11 SMARCA2 CMYA5 NANOG CENPN \\
& ZBTB20 BZW2 GABRG1 GIPC2 WIPF3 \\
\hline
\end{tabular}

Table 4 The GO and KEGG pathway enrichment analysis of the common DEGs 


\begin{tabular}{|c|c|c|c|}
\hline Category & Term & Count & PValue \\
\hline \multirow[t]{5}{*}{ Up-bp } & GO:0051301 cell division & 6 & $\begin{array}{r}0.012861854 \text { NDE1, CCND1, ZWINT, } \\
\text { TPX2, CABLES1, SMC4 }\end{array}$ \\
\hline & $\begin{array}{l}\text { GO:0030855 epithelial cell } \\
\text { differentiation }\end{array}$ & 3 & 0.031824814F11R, COL4A1, VEGFA \\
\hline & GO:0007155 cell adhesion & 6 & $\begin{array}{c}0.036156599 \text { LAMA2, F11R, MAGI1, } \\
\text { CDON, DST, CDH6 }\end{array}$ \\
\hline & GO:0070997 neuron death & 2 & 0.043037304 MAGI1, SLC25A27 \\
\hline & $\begin{array}{l}\text { GO:0030198 extracellular matrix } \\
\text { organization }\end{array}$ & 4 & $\begin{array}{l}0.04353266 \text { LAMA2, F11R, COL4A1, } \\
\text { HPSE }\end{array}$ \\
\hline \multirow[t]{11}{*}{ Up-cC } & GO:0005737 cytoplasm & 33 & $\begin{array}{r}0.001755894 \text { BID, DZIP3, NUAK2, } \\
\text { PNISR, CXXC5, NAA38 }\end{array}$ \\
\hline & $\begin{array}{l}\text { GO:0015630 microtubule } \\
\text { cytoskeleton }\end{array}$ & 5 & $\begin{array}{l}0.001909151 \text { F11R, SHMT2, TPX2, } \\
\text { STRBP, DST }\end{array}$ \\
\hline & $\begin{array}{l}\text { GO:0070062 extracellular } \\
\text { exosome }\end{array}$ & 19 & $\begin{array}{c}0.017512272 \text { BID, F11R, SHMT2, MYO6, } \\
\text { SLC44A2, SUSD2 }\end{array}$ \\
\hline & GO:0005905 clathrin-coated pit & 3 & 0.018944536CTTN, MYO6, SORT1 \\
\hline & $\begin{array}{l}\text { GO:0005765 lysosomal } \\
\text { membrane }\end{array}$ & 5 & $\begin{array}{c}\text { 0.021130923 SLC44A2, MYO6, HPSE, } \\
\text { SORT1, DNAJC5 }\end{array}$ \\
\hline & GO:0005634 nucleus & 30 & $\begin{array}{c}0.024137441 \text { NUAK2, NAA38, N4BP2L2, } \\
\text { CHD7, CDKN2A, HPSE }\end{array}$ \\
\hline & GO:0031012 extracellular matrix & 5 & $\begin{array}{c}0.027087534 \text { LAMA2, COL4A1, CDON, } \\
\text { CFL1, PFKP }\end{array}$ \\
\hline & $\begin{array}{l}\text { GO:0005604 basement } \\
\text { membrane }\end{array}$ & 3 & 0.037063904 LAMA2, COL4A1, DST \\
\hline & GO:0016020 membrane & 15 & $\begin{array}{c}0.038339404 \text { BID, MYO6, PFKP, PSD4, } \\
\text { NDE1, CCND1, HPSE }\end{array}$ \\
\hline & GO:0032587 ruffle membrane & 3 & 0.03966008 MYO6, CFL1, AIF1L \\
\hline & $\begin{array}{l}\text { GO:0000777 condensed } \\
\text { chromosome kinetochore }\end{array}$ & 3 & 0.044135433CENPO, NDE1, ZWINT \\
\hline \multirow[t]{4}{*}{ Up-mf } & GO:0005515 protein inding & 48 & $\begin{array}{r}0.004872107 \text { BID, NUAK2, DZIP3, } \\
\text { SUSD2, CXXC5, PDIA4 }\end{array}$ \\
\hline & $\begin{array}{l}\text { GO:0051015 actin filament } \\
\text { binding }\end{array}$ & 4 & $\begin{array}{l}0.016828637 \text { MYO6, ARPC5L, CFL1, } \\
\text { AIF1L }\end{array}$ \\
\hline & $\begin{array}{l}\text { GO:0008022 protein C-terminus } \\
\text { binding }\end{array}$ & 4 & $\begin{array}{c}0.038467386 \text { EFHC1, MAGI1, TOP2A, } \\
\text { DST }\end{array}$ \\
\hline & GO:0019899 enzyme binding & 5 & $\begin{array}{c}0.04731798 \text { N4BP2L2, CCND1, STC2, } \\
\text { SORT1, TOP2A }\end{array}$ \\
\hline Pathwa & hsa 05200:Pathways in ca & 9 & LAMA2, BID, CCDC6, \\
\hline
\end{tabular}


CCND1, CDKN2A, COL4A1

hsa 05219:Bladder cancer $\quad 30.015355087$ CCND1, CDKN2A, VEGFA

hsa 05212:Pancreatic cancer 30.036353975 CCND1, CDKN2A, VEGFA

hsa 04115:p53 signaling pathway 30.03842294 BID, CCND1, CDKN2A

Down-bp GO:0007399 nervous system development

$40.010670453 \mathrm{BZW} 2$, SMARCA2, FGF2,

GO:0030154 cell differentiation

4 0.037116087BZW2, NANOG, WIPF3, FOXN3

\section{Figures}


A

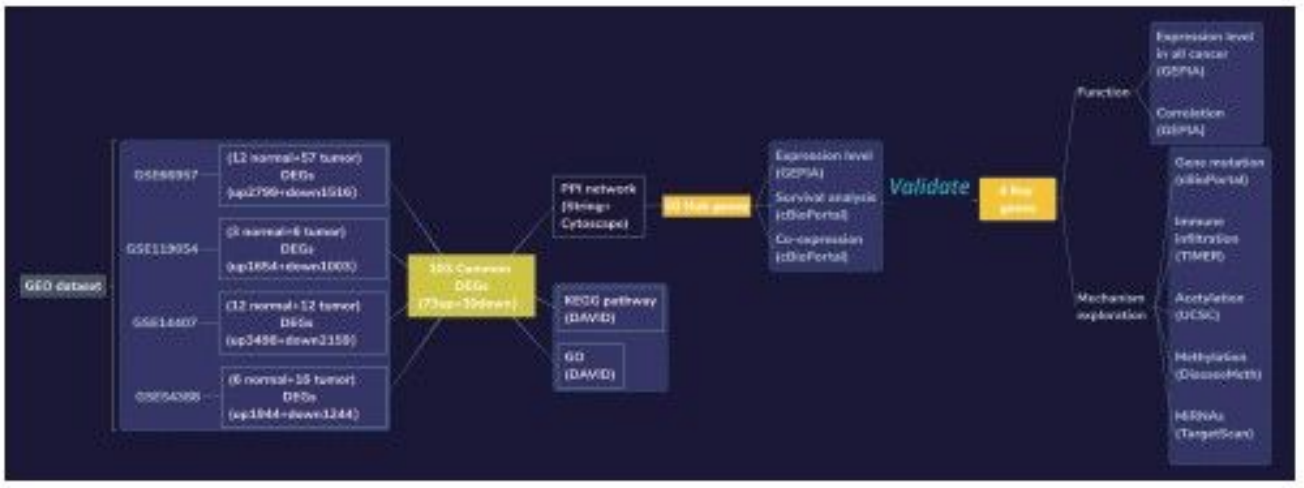

B
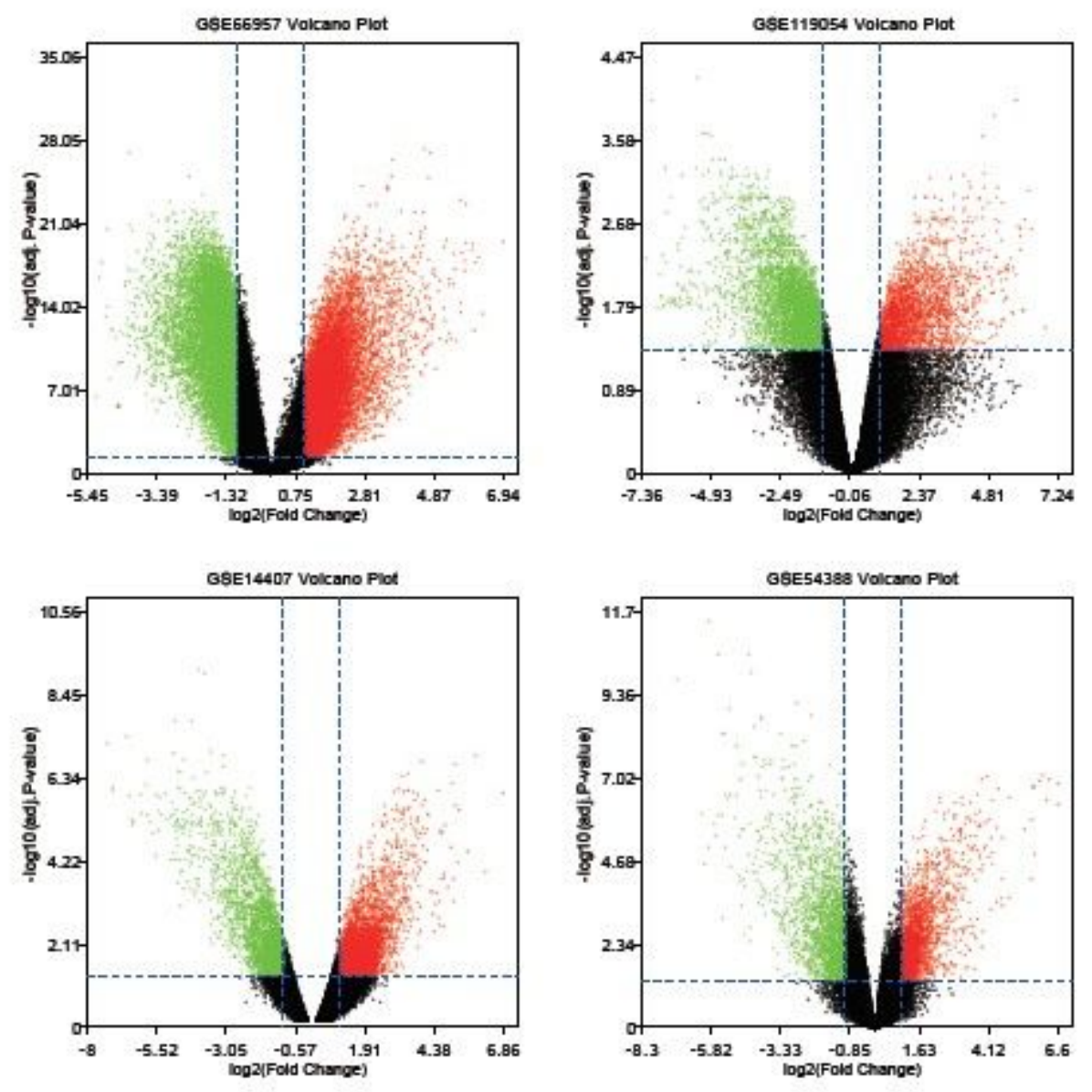

Figure 1

Workflow of the study and the expression of genes between two sets of samples (Cancer VS. Normal) 


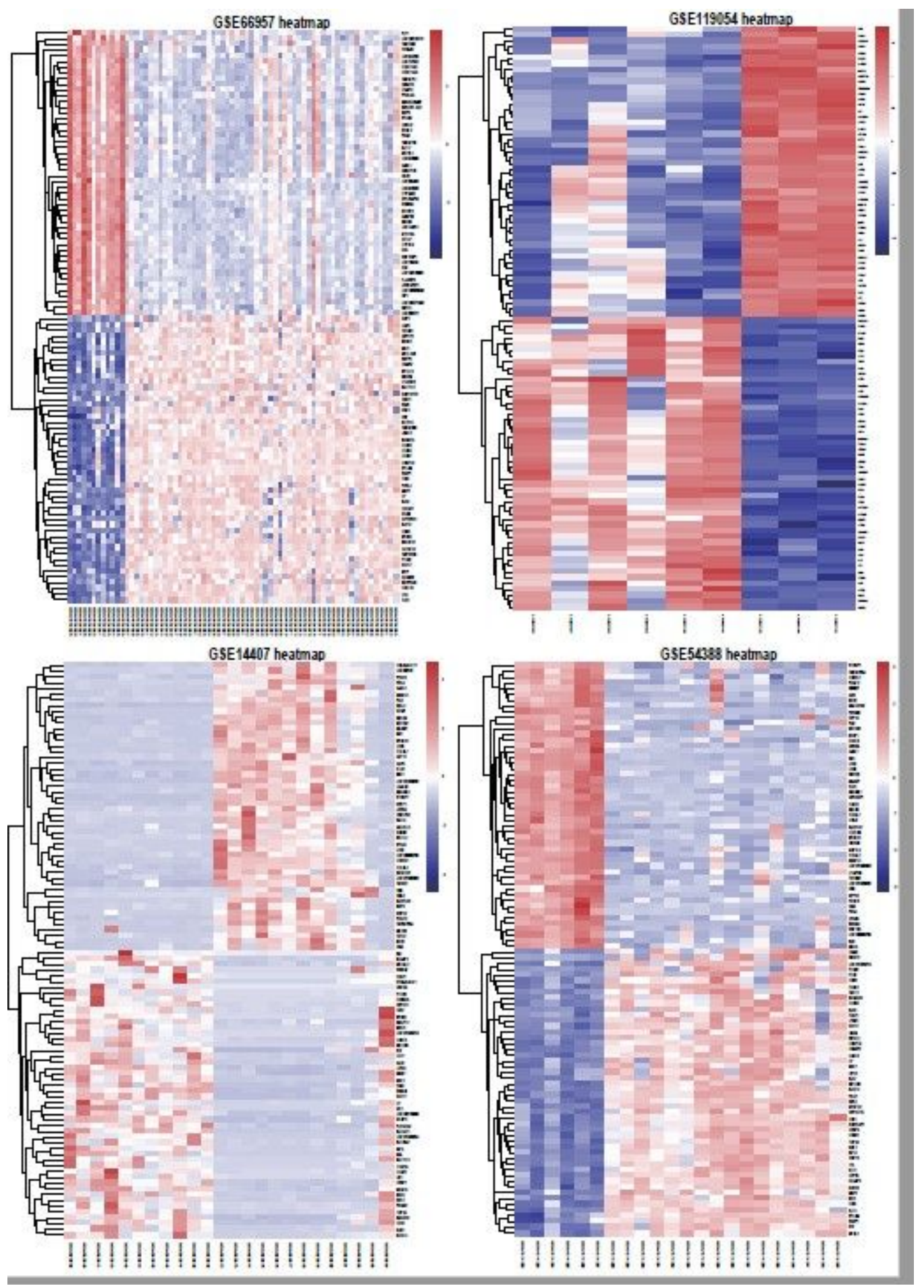

Figure 2

Hierarchical clustering heatmap of DEGs Notes: Hierarchical clustering heatmap of DEGs screened on the basis of |fold change|『2.0 and a corrected adjusted P-value $₫ 0.05$. Firebrick indicates that the relative expression of genes is up-regulated, navy indicates that the relative expression of genes is downregulated. 
A

B
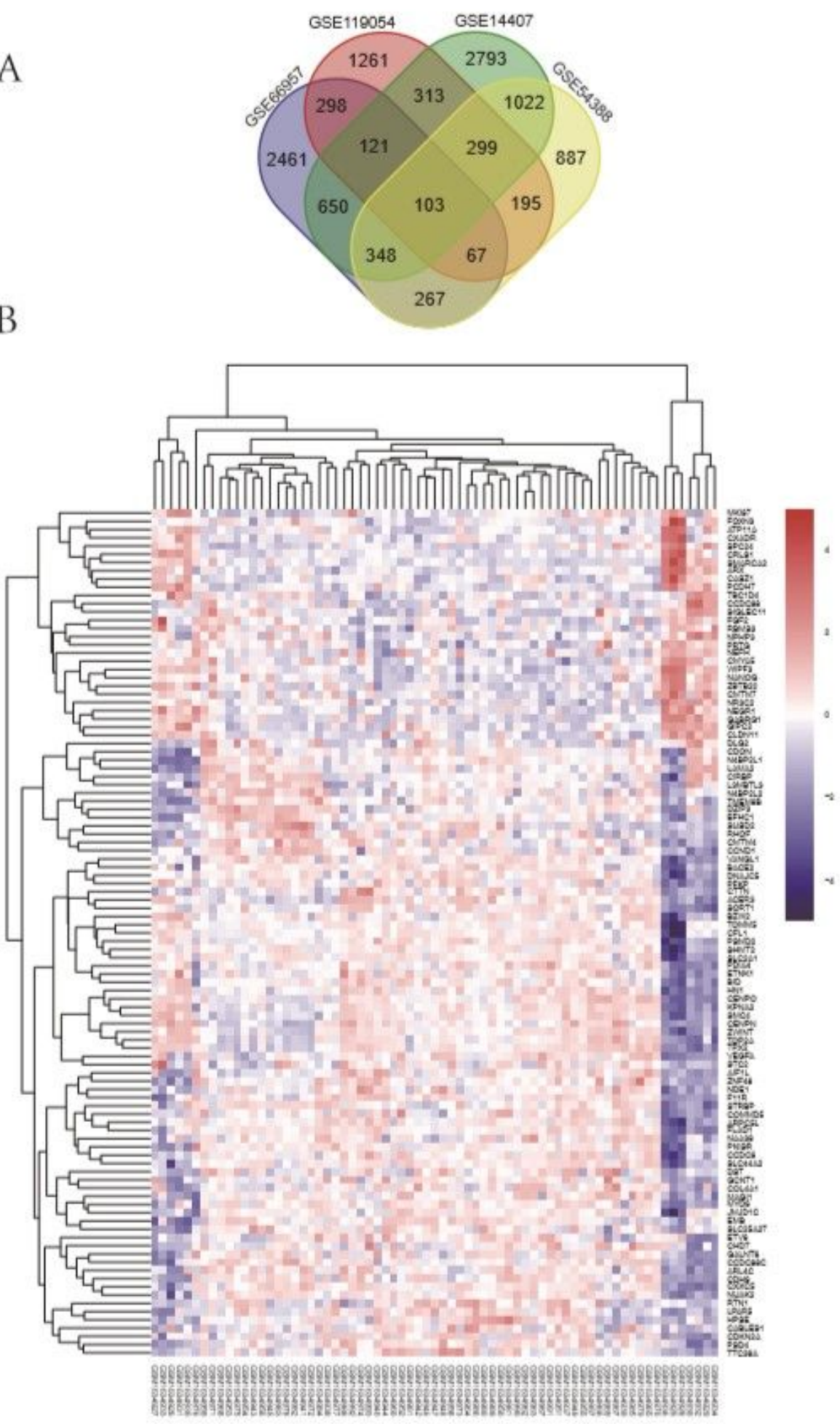

Figure 3

Intersection and Hierarchical clustering heatmap of common DEGs Notes: (A) Overlapping genes of 4 GEO databases including GSE66957, GSE119054, GSE14407 and GSE54388. (B) Hierarchical clustering heatmap of common DEGs. Firebrick indicates that the relative expression of genes is up-regulated, navy indicates that the relative expression of genes is down-regulated. 

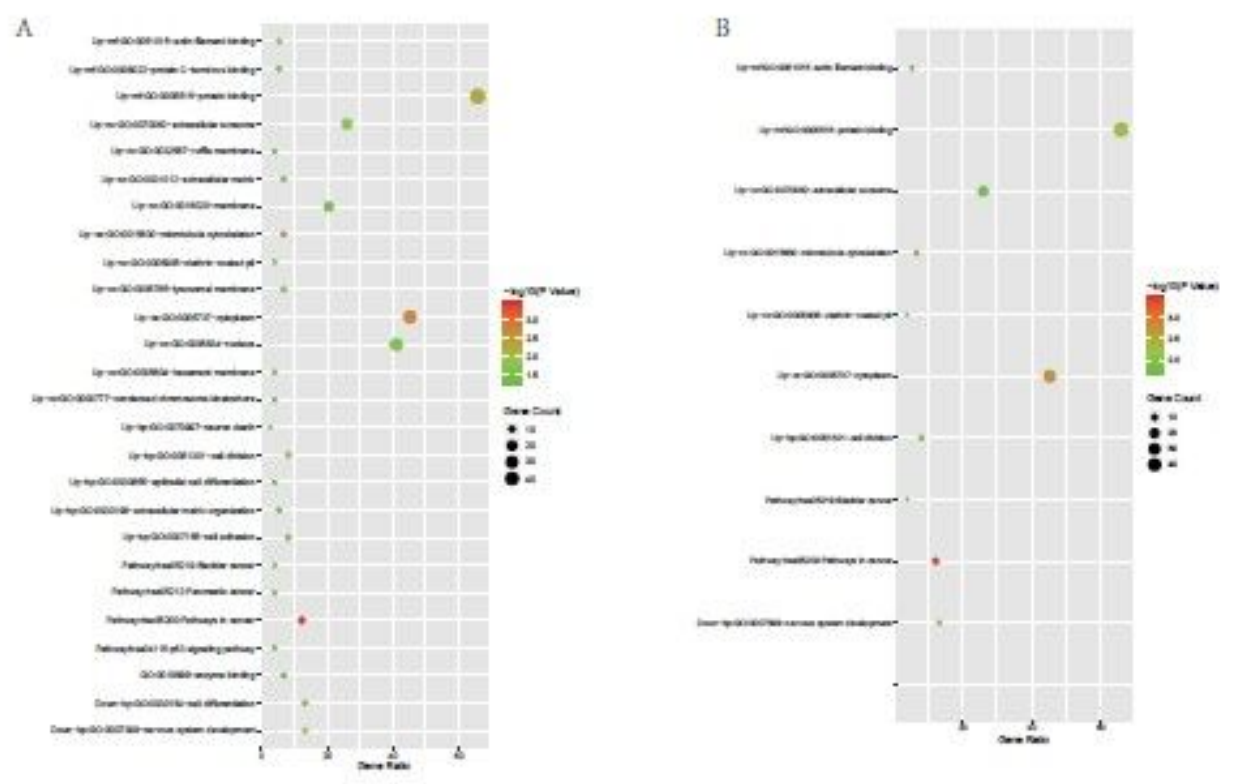

\section{Figure 4}

GO and Pathway enrichment analysis of common DEGs Notes: Enrichment analysis of GO and KEGG pathway and significant enrichment of differentially expressed genes (DEGs) in OC. (A) GO and KEGG pathway analysis was used to classify the common DEGs into BP, CC, MF and pathway group. (B) Top 10 significantly enriched GO and KEGG pathway terms of common DEGs. 


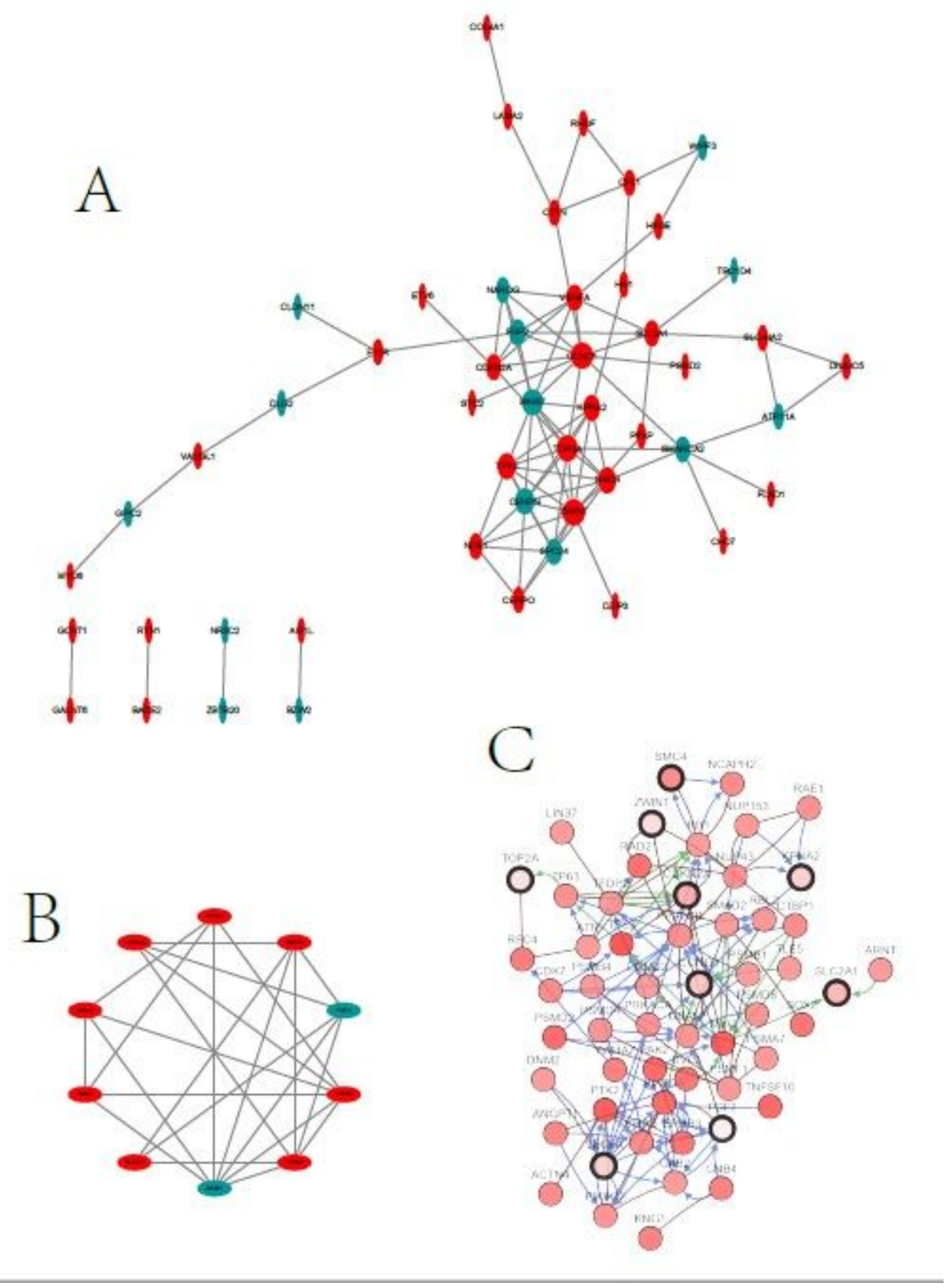

Figure 5

PPI and Hub genes network Notes: (A)The PPI network of common DEGs were constructed using Cytoscape. Circles represent genes, lines represent the interaction of proteins between genes, and the results within the circle represent the structure of proteins. Line color represents evidence of the interaction between the proteins. (B) Top 10 Hub Gene Networks calculated by the Degree Algorithm in the CytoHubba Plugin. The redder the color, the more important the relationship is. (C) Co-expresssion and network of Hub genes. Nodes with bold black outline represent Hub genes. Nodes with thin black outline represent the co-expression genes. 


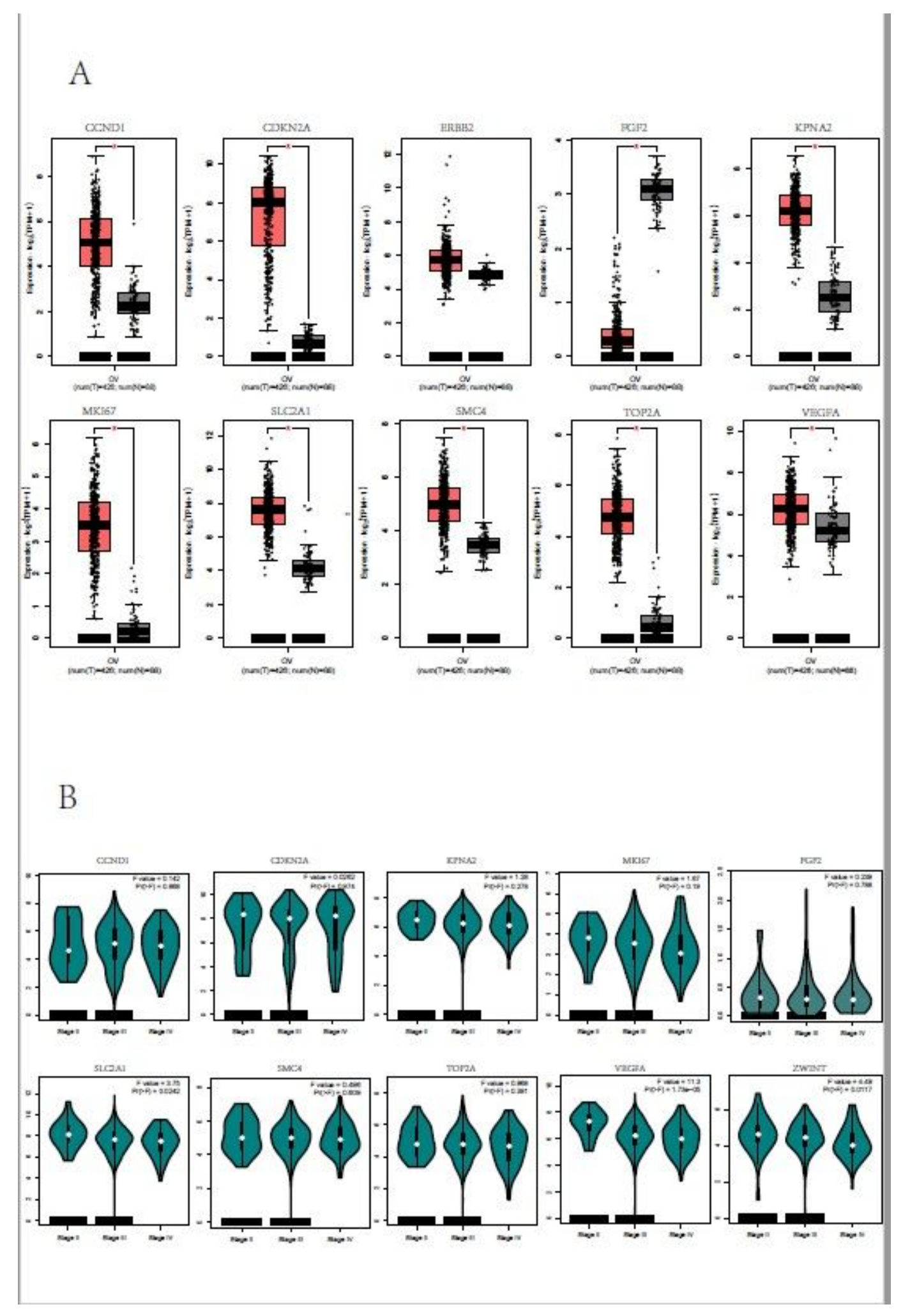

Figure 6

Expression of Hub Genes in $\mathrm{OC}$ and its different grades Notes $\mathbb{( A )}$ Comparison of expression level of Hub genes in ovarian cancer and normal tissues. (B) Comparison of expression level of Hub genes in different grades of ovarian cancer. 

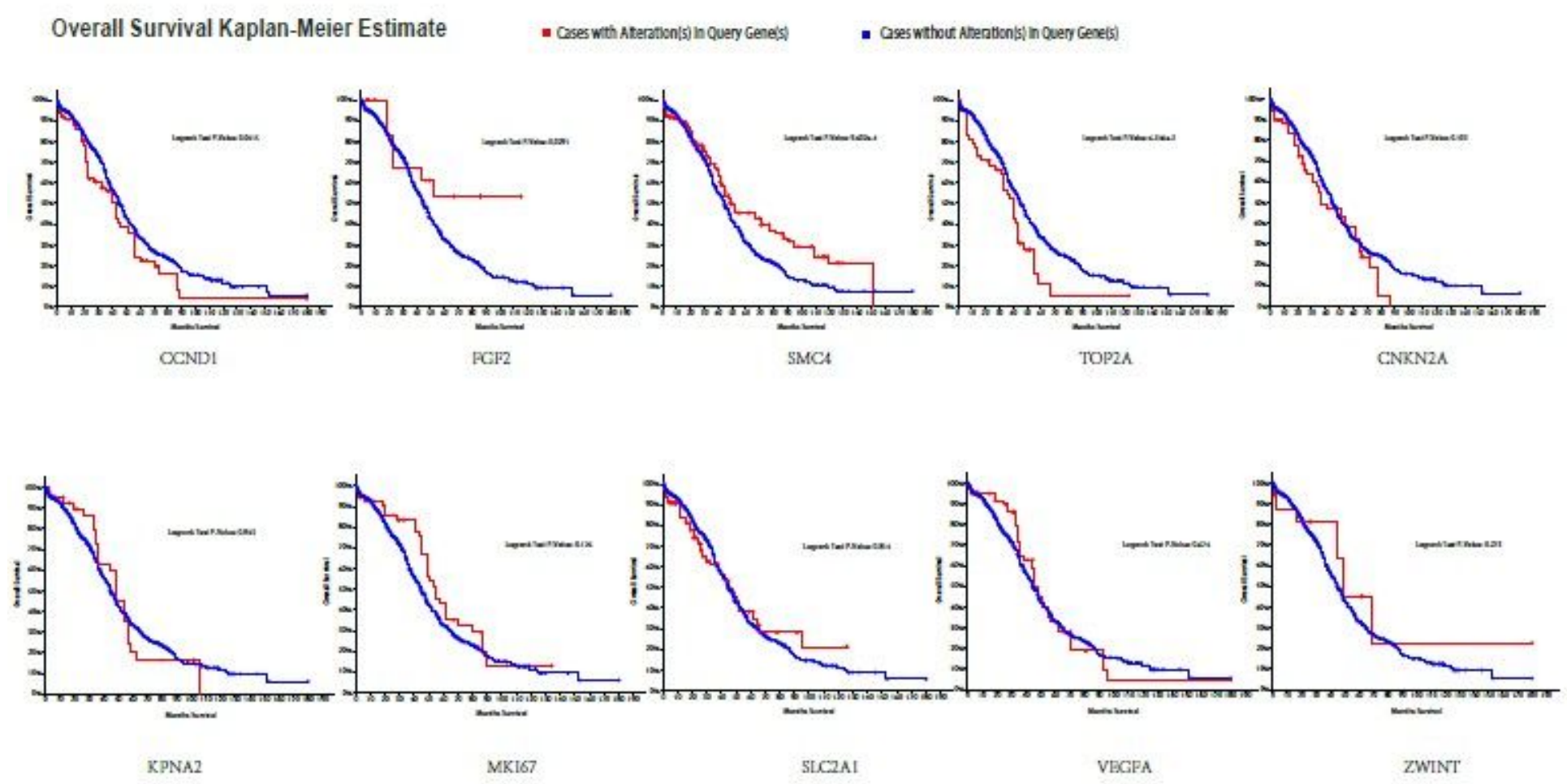

\section{Figure 7}

Total survival of $O C$ with Hub genes Notes: Overall survival analyses of Hub genes were performed using cBioPortal online platform. $\mathrm{P}<0.05$ was considered statistically significant. 


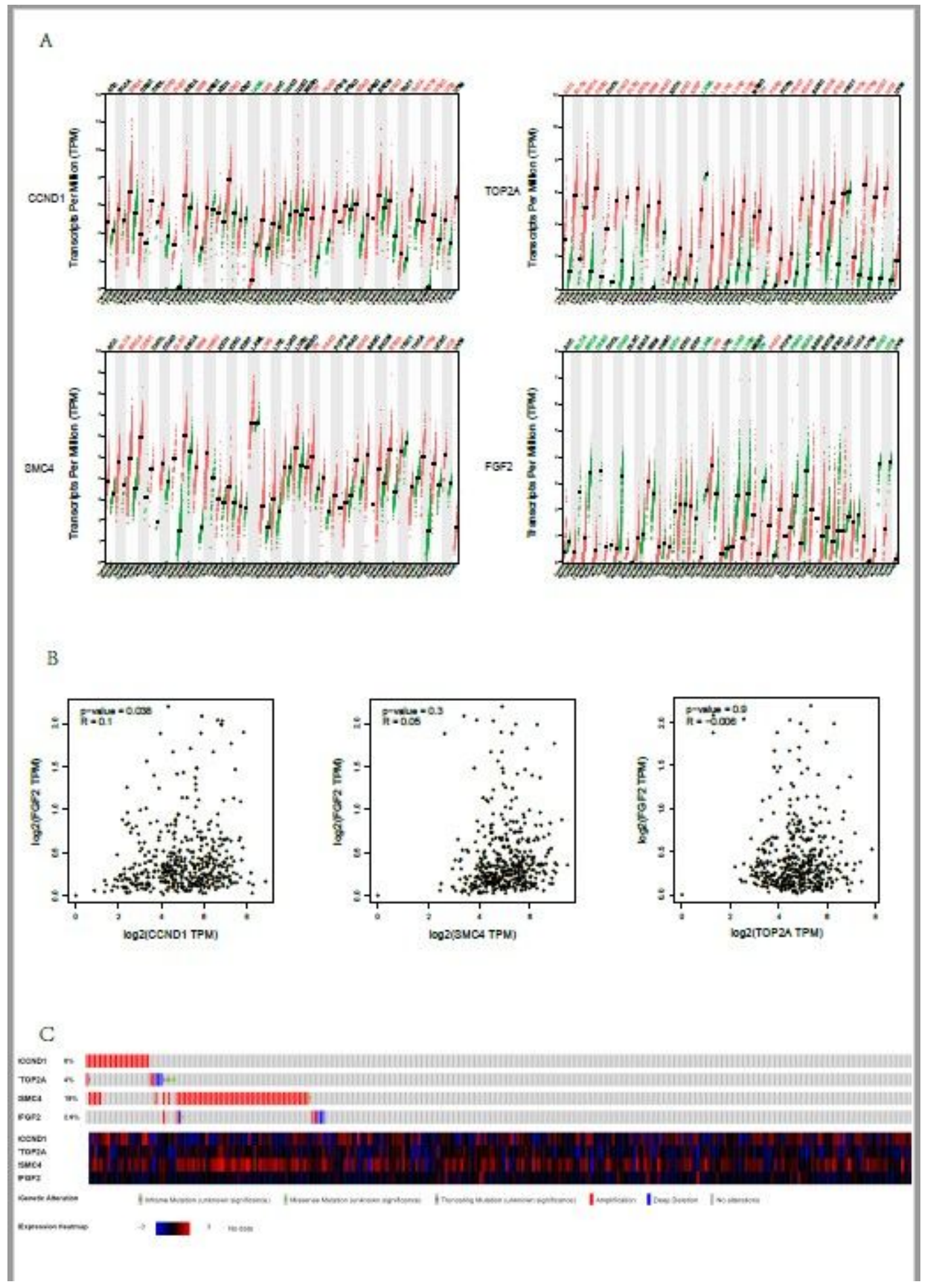

Figure 8

Function of key candidate genes and its regulation mechanism of OC (A) Expression of key candidate genes in multiple tumors. Red represents high expression, green represents low expression. (B) The relationship between fibroblast growth factor 2 and the other three genes. $\mathrm{R}$ represents the degree of correlation, $\mathrm{P}$-value $\mathbb{0 . 0 5}$ is statistically significant. (C) The proportion of gene mutation types and gene cluster expression of Hub genes in $\mathrm{OC}$. 


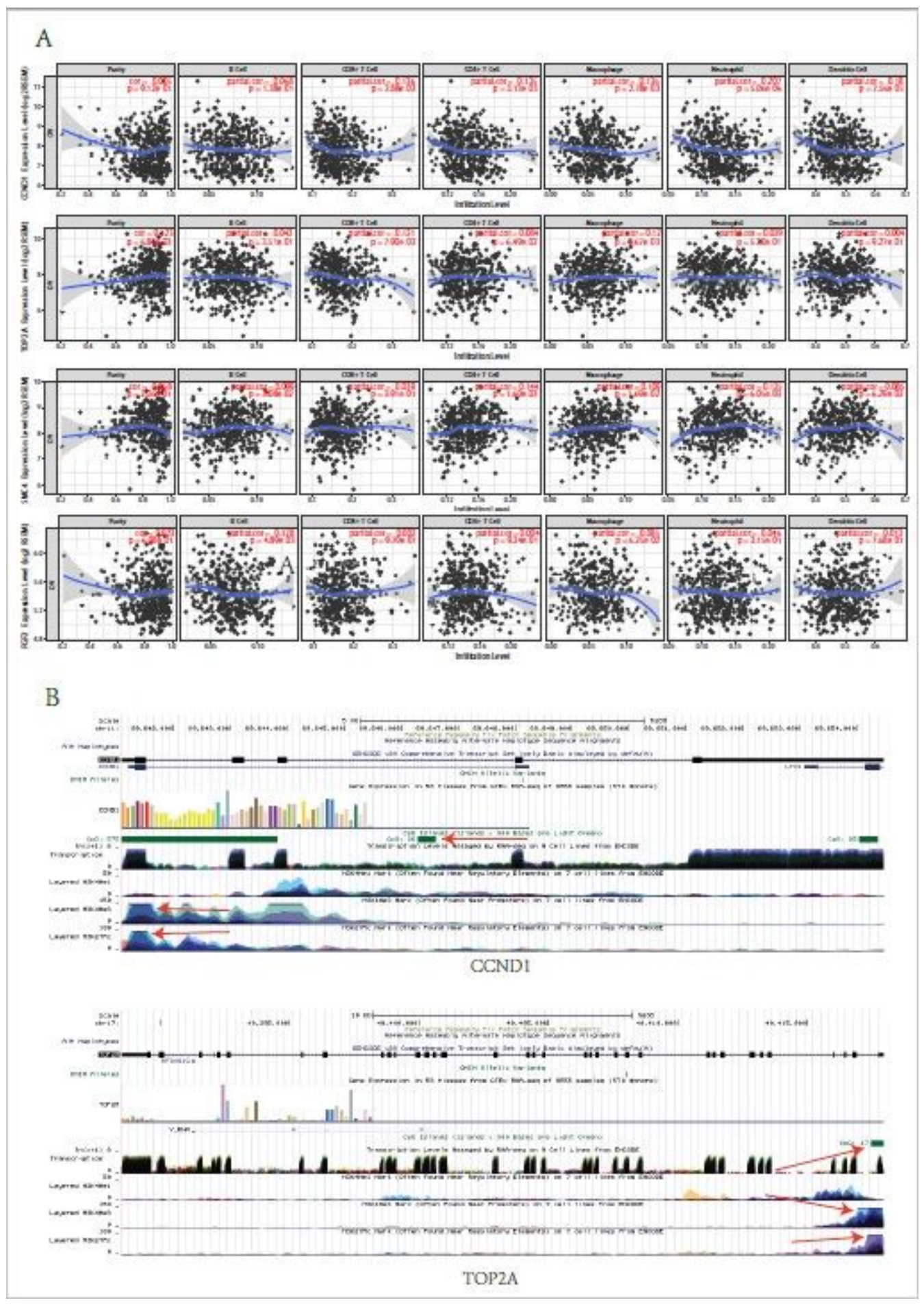

Figure 9

Immune infiltration and epigenetic regulation of key genes (A)The relationship among four key genes and tumor immune infiltration in ovarian cancer. (B) CCND1 and TOP2A sequences have methylation sites and acetylation regulation. Arrows refer to methylation and acetylation sites. 


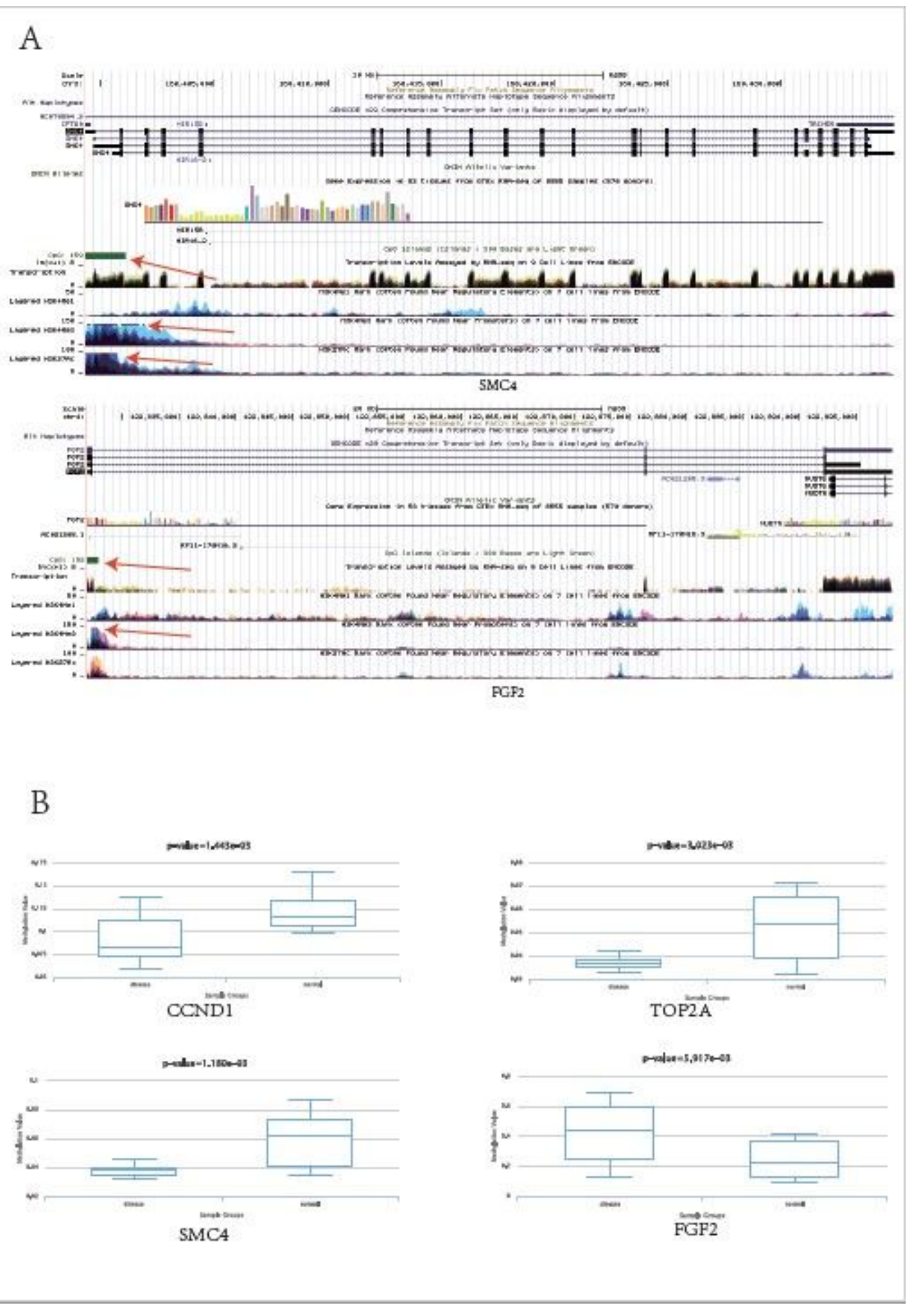

Figure 10

Epigenetic regulation and methylation of key genes Notes:(A) SMC4 and FGF2 sequences have methylation sites and acetylation regulation. Arrows refer to methylation and acetylation sites. (B)Methylation levels of four genes in $\mathrm{OC}$ and normal tissues. 


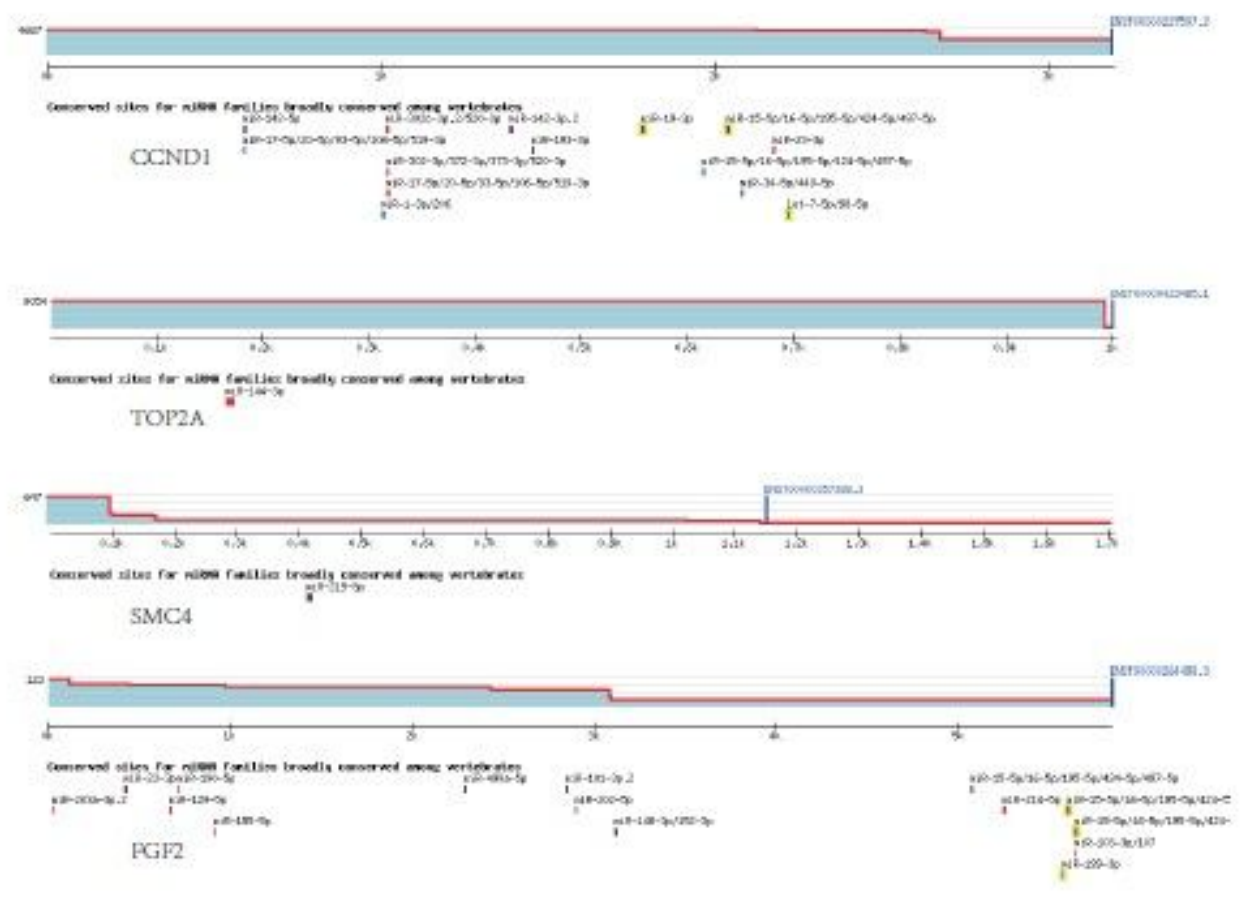

\section{Figure 11}

The binding sites of key genes to miRNAs. Notes: Each gene sequence has multiple miRNAs binding sites. 